\title{
Minimal requirements for robust cell size control in eukaryotic cells
}

\author{
Benjamin Pfeuty and Kunihiko Kaneko \\ Department of Pure and Applied Sciences, University of Tokyo, Tokyo 153-8902, Japan \\ E-mail: pfeuty@complex.c.u-tokyo.ac.jp
}

Received 13 July 2007

Accepted for publication 10 September 2007

Published 9 October 2007

Online at stacks.iop.org/PhysBio/4/194

\begin{abstract}
Most cell types living in a stable environment tend to keep a constant characteristic size over successive generations. Size homeostasis requires that cells exert a tight control over the size at which they divide. Cell size control is not only robust against various noises, but also highly flexible since cell sizes can vary tremendously, notably as a function of nutrient levels. We formulated a minimal mathematical model of the eukaryotic cell cycle in which the cell size control operates through a cell growth-dependent bifurcation in the cell cycle dynamics. Such a bifurcation mechanism can readily explain the occurrence of a minimum critical size at division under limiting growth conditions. However, it also predicts that cells should become progressively larger and larger under prolific growth conditions. We argue that the cell size control can be reinforced at fast growth rates by adding a new cell cycle inhibitory activity whose strength would increase with the cell growth rate. We further show that various sources of noise may also generate a large variability in cell size at division and interdivision time that exhibit characteristic exponential tail distributions, without compromising the robustness of the cell size control.
\end{abstract}

\section{Introduction}

Most proliferating cell types that grow in a stable environment tend to display a constant characteristic size over successive generations. Size homeostasis requires that cells born with a standard size double their size during interdivision time while daughter cells born with a size above (or below) average would divide before (or after) they reach twice their size. By inference, interdivision time should be shorter for oversized newborn cells than for small newborn cells. Size homeostasis is observed in a wide variety of cells including bacteria [1], budding yeast [2], fission yeast [3], Xenopus laevis [4], Amoeba [5], Chlamydomonas [6] and mammalian cells [7]. In order for cell size homeostasis to be achieved, a mechanism of coordination between the cell growth and cell division is necessary. Such a coordination process appears to primarily rely on the requirement that cells should not commit to divide before they attain a rate of macromolecular synthesis, reflected by a certain size, that is sufficient to perform quickly, safely and autonomously the major cell cycle (CC) events [8]. Therefore, the CC can be divided into two successive phases: (i) a sizer phase that corresponds to the time period necessary to reach the critical cell size meeting the above requirement and that depends on the cell size at birth, and (ii) a timer phase that corresponds to the time period necessary to initiate the duplication process and that is nearly independent of the birth size $[3,9,10]$.

Cell size control, however, is not rigidly programmed. It is rather flexible since the cell sizes can vary over a wide range, notably as a function of nutrient levels [11-14]. Furthermore, cells growing under the same nutrient conditions generally display a significant though limited size variation [15-17]. Strikingly, interdivision time distributions are broader than critical cell size distributions and typically display an exponential tail [3,16-18]. Various sources of noise could give rise to the experimentally observed variability in cell size and interdivision time in steady state populations of wild-type cells: e.g., noise in the molecular dynamics [19], occurrence of asymmetric divisions [20] and heterogeneity in the growth rate of co-living cells [19].

Mathematical modeling and nonlinear dynamics have been essential tools to gain insights into the dynamics of the CC molecular network [21-23]. Regarding cell size homeostasis, cell size checkpoints are viewed as bifurcations 
in the dynamics of the $\mathrm{CC}$ molecular network, in which the size (referring to either the cell volume or the cell mass) is the main bifurcation parameter [10,21]. It can be questioned, however, to which extent the cell size control in these models is robust in the presence of noises or against environmental changes. In these models, indeed, the cell size at division could be sensitive to the cell growth rate [24] and to a variety of noises [25].

Since most cells share similar cell size control features despite the expected disparities between their intracellular biochemical reaction networks, it can be anticipated that the mechanisms of coordination between their growth and division processes share the same core dynamics. Here, we formulate a minimal mathematical model of the eukaryotic $\mathrm{CC}$ that could serve as a framework to study the generic properties of the cell size control. The model postulates an exponential growth dynamics coupled with a two-variable CC network dynamics. Combining analytical and numerical approaches, we have investigated how cell sizes at division and interdivision times depend on (i) kinetics of the biochemical reactions, (ii) cell sizes at birth, (iii) cell growth rates and (iv) noise levels. One of the dynamical scenarios that could give rise to cell size control is based on the hypothesis that increasing the cell volume triggers a saddle-node infinite period bifurcation (SNIPER) in the dynamics of the CC network [24]. As stressed by the authors, however, such dynamical behavior would lead to an increase in cell size at division with increasing growth rates and a concomitant weakening of cell size control. We show here that the cell size control can be reinforced at fast growth rates by adding a new growth-rate-dependent mechanism of $\mathrm{CC}$ regulation. We further analyze how stochastic processes in the dynamics of the $\mathrm{CC}$ molecular network and of cell growth affect the variability in critical cell size and interdivision time. We show that both sources of noise account for the exponentiallike distributions of these variables that are actually observed.

\section{Mathematical modeling}

During its division cycle, a cell must sequentially replicate its genome and segregate its two sets of chromosomes during mitosis before splitting into two and giving birth to twin sisters. These events that are triggered by an interacting set of specific molecular components define the $C C$ dynamics. At the same time, proliferating cells need most of the time to synthesize a diversity of cell components other than the $\mathrm{CC}$ regulatory components, especially the central building units of the protein-synthesis apparatus, i.e. the ribosomal proteins. This parallel activity that is reflected by an overall cell growth defines the cell growth dynamics. We propose here a minimal mathematical model that couples the dynamics of cell growth and of the $\mathrm{CC}$ regulatory components (figure 1).

\subsection{Cell growth dynamics}

Individual cell growth (i.e., cell volume or cell mass expansion) principally results from the intracellular biosynthetic activity sustained by resources harvested from the cell environment.

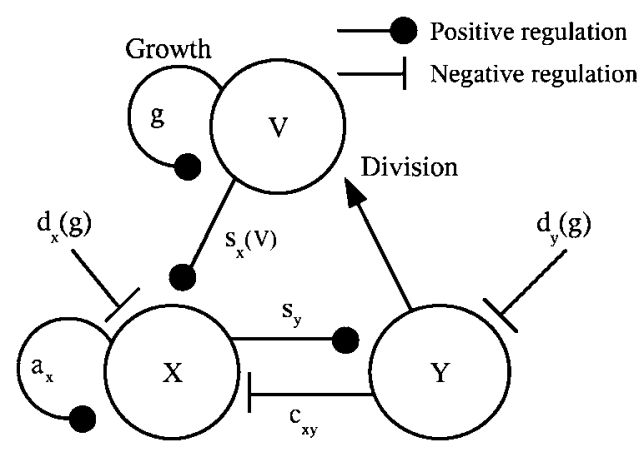

Figure 1. Schematic representation of the model. The model links the dynamics of cell volume growth, $V$, to the dynamics of two sets of interacting $\mathrm{CC}$ regulatory components, $X$ and $Y$.

Therefore, its dynamics depends on specific characteristics of both the cell and its environment and may exhibit various patterns (e.g., linear or exponential ones) depending on the particular setting. Different cell growth patterns can even be obtained depending on the method of measure (cell volume or dry mass measurement) [26]. In addition, it is extremely difficult to distinguish between linear and exponential growth over a single doubling time [27, 28]. Thus, it has been reported that the fission yeast displays a bilinear growth pattern $[3,27]$ while the budding yeast grows exponentially [19, 29, 30]. In this work, we choose to assume that cell growth is exponential essentially because (i) this approximation appears to be a reasonable one in many cases; (ii) it is easy to rationalize (mass synthesis is proportional to the cell mass) and (iii) it is often used in modeling studies:

$$
\frac{\mathrm{d} V_{i}}{\mathrm{~d} t}=g_{i} V_{i}
$$

We postulate that different cells $i$ may have different growth rates, $g_{i}$, normally distributed with a mean, $g$, and a standard deviation, $\sigma_{g}$.

\subsection{Cell cycle dynamics and division}

Dynamical models of eukaryotic CC require at least two variables [31-33]. The first variable is a set of components (called $X$ ) involved in initiating a cascade of reactions that trigger the major $\mathrm{CC}$ events. Examples of $X$-components are the activated cyclin-Cdk (Cyclin-dependent kinase) complexes in eukaryotic cells [34]. The second variable is a set of components (called $Y$ ) that inhibit the activity of $X$-components and trigger the physical process of division (cytokinesis). The APC (anaphase promoting complex) in conjunction with Cdc20 and Cdh1 is supposed to fulfil this function in eukaryotic cells [35].

The detailed dynamics of a two-variable system can take various mathematical forms. For the sake of simplicity, we focus on the dynamics of protein synthesis and degradation rather than of phosphorylation and dephosphorylation $[33,36]$. The concentrations of the $X$ - and $Y$ - components vary with time as follows:

$$
\begin{gathered}
\frac{\mathrm{d} X}{\mathrm{~d} t}=s_{x}-\left(d_{x}+c_{x y} Y\right) X+a_{x} X^{2}+\sigma_{x} \zeta_{x}(t) \\
\frac{\mathrm{d} Y}{\mathrm{~d} t}=-d_{y} Y+s_{y} X^{2}+\sigma_{y} \zeta_{y}(t) .
\end{gathered}
$$


The $X$-components activate the $Y$-components (with a rate constant $s_{y}$ ) and possess an autocatalytic activity (with a rate constant $a_{x}$ ), which is likely to result from positive feedback loops caused by the mutual inhibition with other components. The $Y$-components, in turn, repress the activity of the $X$-components with a rate constant $c_{x y}$. The concentrations of the $X$ - and $Y$-components decrease with a rate constant $d_{x}$ or $d_{y}$ (through either degradation, inactivation or dilution).

The stochastic nature of the biochemical reactions leading to the statistical fluctuations in the concentration of molecular components is modeled by a Gaussian white noise $\zeta_{\alpha}(t)$ with a zero mean and standard deviation $\sigma_{\alpha}(\alpha=x$ or $y)$ which depends on the concentrations of $X$ - and $Y$-components. Indeed, if the number of components is much larger than unity and chemical reaction events take place in time as Poisson processes, the noise amplitudes $\sigma_{\alpha}$ are given by [37]

$$
\begin{gathered}
\sigma_{x}^{2}=\eta^{-1}\left(d_{x} X+c_{x y} Y X+a_{x} X^{2}+s_{x}\right) \\
\sigma_{y}^{2}=\eta^{-1}\left(d_{y} Y+s_{y} X^{2}\right)
\end{gathered}
$$

The prefactor $\eta$ corresponds to the number of molecular components whose concentration is equal to 1 in a cell volume $V=1$ in our dimensionless units.

In the model, we assume that cytokinesis takes place at time point $\tau$ when the $Y$-component concentration reaches a critical value $\theta$. The size of daughter cells is normally distributed with a mean, $V(\tau) / 2$, a standard deviation, $\sigma_{d}$, and a coefficient of variation, $c_{v, d}=2 \sigma_{d} / V(\tau)$. We further assume that, along successive cell divisions, $c_{v, d}$ is kept fixed but not $\sigma_{d}$. Hereafter, the variables $V_{b}$ and $V_{d}$ will refer to the cell volumes at birth and at division, respectively, of different cells. The variable $T$ will refer to the interdivision time between cell birth and cell division.

\subsection{Coupling between growth and division}

The dynamics of the CC regulatory components and of cell growth can be coupled in two opposite ways. For the sake of simplicity, we solely consider the case where the cell growth can modify the rate of accumulation and/or activation of the $\mathrm{CC}$ regulatory components. Importantly however, we postulate that the cell size and cell growth rate exert a differential effect on CC dynamics. First, the cell growth leads to a dilution of the chemical concentrations within cells. Hence, the activity of $\mathrm{CC}$ regulatory components decreases at a speed, $d_{\alpha}$, equal to the sum of their overall rate of disappearance, $d_{\alpha, 1}$, and of the rate of cell growth $g$ :

$$
d_{\alpha}=d_{\alpha, 1}+g, \quad \alpha=(x, y) .
$$

It is generally assumed that the rate of appearance of the active cyclin-Cdk complexes is an increasing function of the cell size. Because cyclins are synthesized and bind to their $\mathrm{Cdk}$ partner in the cytoplasm before the complexes are transported and activated in the nucleus [38], the rate of cytoplasmicnuclear transport of the cyclin-Cdk complexes is likely to be a limiting factor in the rate of appearance of the active cyclinCdk complexes [39]. Such a transport rate, which depends on the ratio between the cytoplasmic volume and nuclear surface and, thereby, on the cell size, can be qualitatively modeled by considering that the rate of appearance of the $X$-components increases linearly with the cell volume:

$$
s_{x}=s_{x, 1}+s_{x, 2} V \text {. }
$$

\subsection{Model parameters}

Since we are interested in qualitative properties of the CC dynamics, it is convenient to use dimensionless variables and parameters. $d_{x, 1}=d_{y, 1}=1$ indicates that the timescale unit of the system coincides with the rate of disappearance of the CC regulatory components. The parameters $c_{x y}=2$ and $s_{y}=0.5$ are kept constant throughout the work. Changing these parameters does not significantly affect the qualitative results. Nevertheless, the ratio of these parameters is tuned to balance the feedforward excitation and the feedback inhibition and to obtain similar oscillation amplitudes for both $X$ and $Y$ variables in the limit cycle regime. The threshold concentration of the $Y$-components for division is set to $\theta=2$ (unless otherwise stated). We focus on how the $\mathrm{CC}$ dynamics depends on the parameters $g, a_{x}, s_{x, 1}, s_{x, 2}, \eta, \sigma_{g}$ and $c_{v, d}$.

\section{Results}

\subsection{Cell cycle dynamics at a fixed cell volume}

A preliminary step before studying the coupling between the dynamics of the CC network and of cell growth consists of analyzing the dynamics of the $\mathrm{CC}$ regulatory components separately, at a fixed cell volume and with the standard deviation of noise set to zero. We focus on the solutions of the dynamical system (2), either a fixed point or a limit cycle (i.e., periodic behavior). The fixed points $\left\{X_{0}, Y_{0}\right\}$ of the $\mathrm{CC}$ dynamics satisfies the following equations:

$$
\begin{gathered}
-\frac{c_{x y} s_{y}}{d_{y}} X_{0}^{3}+a_{x} X_{0}^{2}-d_{x} X_{0}+s_{x}=0 \\
Y_{0}-s_{y} / d_{y} X_{0}^{2}=0 .
\end{gathered}
$$

The linear stability of the fixed points is evaluated by calculating the eigenvalues of the Jacobian matrix of the dynamics. The stability of the fixed point is obtained by the sign of the real parts of the two eigenvalues. A fixed point can be either stable or unstable if both eigenvalues are positive or negative. If one eigenvalue is positive and the other is negative, the fixed point is a saddle point. In a two-dimensional dynamical system, a limit cycle can coexist with fixed points. The existence of a limit cycle is checked numerically.

Figure 2(a) displays the phase diagram depicting the stability of fixed points as a function of the synthesis rate $s_{x}$ and the autocatalytic rate $a_{x}$. The unique fixed point is stable for small enough $a_{x}$ and $s_{x}$. In contrast, when both $a_{x}$ and $s_{x}$ are sufficiently large, the unique fixed point is unstable where a stable limit cycle exists. For small enough $s_{x}$ but sufficiently large $a_{x}$, there is an intermediate parameter region in which three fixed points (stable, unstable and a saddle) are present.

The lines that separate the different dynamical regimes define the parameter values at which a bifurcation occurs. Two 

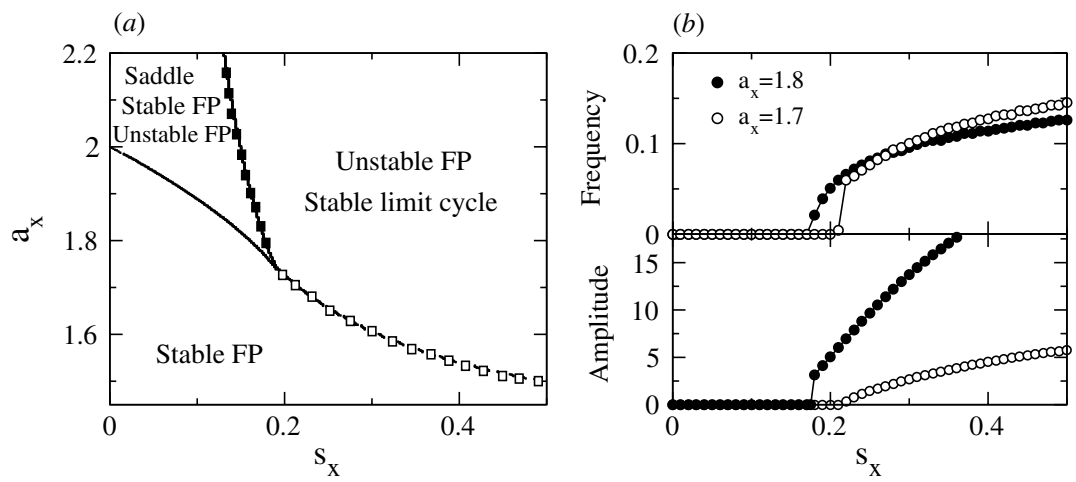

Figure 2. Steady states of the CC dynamics. (a) Bifurcation diagram of the dynamics of CC regulatory components (2) as a function of the rate of appearance of the $X$-components $s_{x}$, and of the autocatalytic rate $a_{x} . F P$ refers to a fixed point. (b) Change in frequency (upper panel) or in amplitude (lower panel) of the concentration of the $X$-components as a function of $s_{x}$. Depending on the value of $a_{x}$, a SNIPER bifurcation $\left(a_{x}=1.8: \bullet\right)$ or a supercritical Hopf bifurcation $\left(a_{x}=1.7: 0\right)$ arises.

types of CC bifurcation, which coincide with the appearance of a stable limit cycle, have been found: (i) a supercritical Hopf bifurcation occurring between a stable and unstable fixed point (open squares) and (ii) a saddle-node infinite period bifurcation (SNIPER) at which two fixed points coalesce on an invariant circle, giving rise to a limit cycle (black squares). The frequency and the amplitude of the limit cycle exhibit properties that are specific to each type of bifurcation. This is illustrated in figure $2(b)$ in which the amplitude and the frequency of the emerging limit cycle are plotted as a function of $s_{x}$ : the SNIPER bifurcation is associated with a continuous increase in frequency and a discontinuous increase in amplitude; in contrast, the supercritical Hopf bifurcation is associated with a discontinuous increase in frequency and a continuous increase in amplitude. The $\mathrm{CC}$ dynamics at a fixed cell volume will serve as a reference to interpret how the cell growth influences the $\mathrm{CC}$ dynamics.

\subsection{Failure of cell size control in the absence of coupling between growth and division}

Because cells grow exponentially, they fail to obey the size homeostasis rule through successive division cycles if there is no coupling between their growth and division processes $\left(s_{x, 2}=0\right)$.

Let us consider the case where the $\mathrm{CC}$ dynamics displays periodic behavior associated with the existence of a stable limit cycle. When the rate of appearance of the $X$-components is sufficiently large, the concentration of the $\mathrm{CC}$ regulatory components oscillates with a period $T_{\mathrm{lc}}$ (figure $2(b)$ ). The ratio between the cell volume at the division of cells generated during two successive cycles $n$ and $n+1$ is given by

$$
V_{d}(n+1) / V_{d}(n)=e^{g T_{\mathrm{lc}}} / 2 \text {. }
$$

If their growth rate $g$ is smaller (or larger) than $T_{\mathrm{lc}} / \ln (2)$, the cells progressively shrink (or swell).

\subsection{How does the coupling between growth and division lead to cell size control}

In order for size homeostasis to occur, it is necessary, although not sufficient, that, through cell volume expansion, the cell growth dynamics modulates the $\mathrm{CC}$ dynamics and, thereby, controls CC progression. Figure 3 illustrates how the cell size control occurs in two distinct ranges of parameters. An asymmetry in cell division with a coefficient of variation of 0.2 has been introduced as an estimate of the strength of the cell size control. The growth rate is kept fixed to $g=0.02$.

Figure 3(a) depicts a first situation in which $s_{x}$ varies in proportion to the cell volume $\left(s_{x, 1}=0, s_{x, 2}=0.1\right)$ and $a_{x}=1.8$. The concentration of the CC regulatory components displays periodic behavior while the cell volume remains bounded along successive divisions (figure $3(a)$ i). The cell volume at division remains roughly constant as the cell volume at birth increases. Consistently, the interdivision time decreases as the cell volume at birth increases (figure 3(a)ii). The dynamical mechanism of cell size control that operates in this case is visualized by plotting the concentration of the $X$-components as a function of the cell volume (figure 3(a)iii). The change in the $X$-component concentration between cell birth and cell division is compared with their steady state concentration, $X_{0}$, computed at fixed volumes from equations (5) and (6). The CC dynamics in cells with different birth volumes (thin full lines) is attracted to the same stable fixed point (thick full line) before crossing a SNIPER bifurcation at identical cell volumes and undergoing similar oscillations around an unstable fixed point (thick broken line). In this case, therefore, cells that display different volumes at birth divide when they reach the same volume. It should be noted that cells in a slightly different situation, where $a_{x}$ is reduced such that the $\mathrm{CC}$ dynamics crosses a supercritical Hopf bifurcation instead of a SNIPER bifurcation, exhibit a strong size control as well (not shown).

Figure $3(b)$ depicts a very different situation where the $\mathrm{CC}$ dynamics is periodic, regardless of cell volume. This occurs when the rate of appearance of the $X$-components exceeds the critical rate at which a SNIPER bifurcation in the underlying $\mathrm{CC}$ dynamics arises (see figure 2). For instance, for $s_{x, 1}=0.18$ and $s_{x, 2}=0.005$, a cell size control still operates because oversized newborn cells exhibit shorter interdivision times than small-sized newborn cells. The cell volume at division, however, is not constant but increases with the cell volume at birth. In this case, the dynamical mechanism of cell 
(a)

(i)

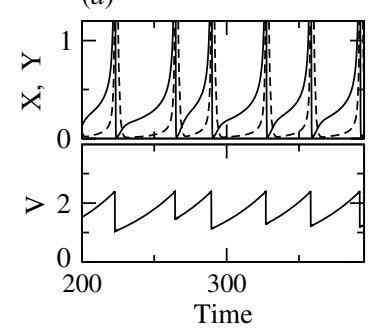

(ii)

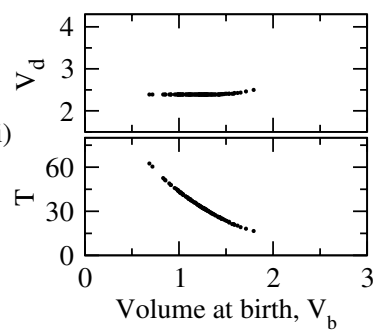

(iii)
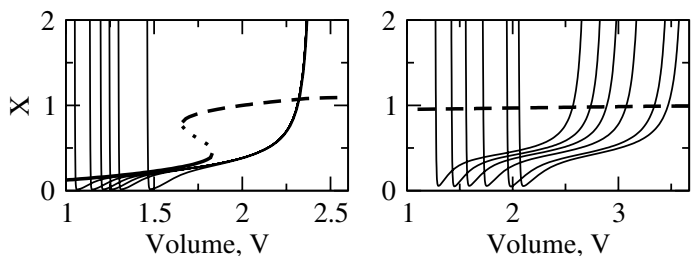

Figure 3. Strong and weak cell size control. Results from simulations of cell proliferation with $g=0.02$ in the presence of a random asymmetry in cell division $\left(c_{v, d}=0.2\right)$. Upper panels: plots of the cell volume and of the concentrations of the $X$ - $Y$-components over successive generation times. Middle panels: plots of the cell volume at division, $V_{d}$, and of the interdivision time, $T$, as a function of the cell volume at birth, $V_{b}$. Bottom panels: change in the $X$-component concentration in a few cells as a function of the cell volume $V$ (thin lines), as compared to their steady state concentration, $X_{0}$ (computed at fixed volumes). The steady state can be stable $(-)$, unstable $(---)$ or a saddle $(\cdots \cdots)$. Parameter values selected: $(a) a_{x}=1.8, s_{x, 1}=0, s_{x, 2}=0.1$ : the CC dynamics exhibits a SNIPER bifurcation. (b) $a_{x}=1.8, s_{x, 1}=0.18$, $s_{x, 2}=0.005$ : the CC dynamics exclusively operates in the limit cycle regime.

size control relies on the fact that the concentrations of $\mathrm{CC}$ regulatory components oscillate with an increasing frequency when the cell volume expands. Such a mechanism works on the condition that the change in frequency with cell volume occurs quickly as is the case here because the system stands close to a SNIPER bifurcation.

\subsection{Fast-growing cells are oversized and display a weak or deficient size control}

The growth rate of individual cells can vary over a wide range depending on a multiplicity of factors, notably the availability of nutrients, the genetic background, the exposure to stresses and to chemical signals from other cells, all of which could impinge on the cell size control mechanism.

Figure 4 illustrates the properties of cell size control in a situation similar to that of figure $3(a)$, except that the growth rate has been raised up from $g=0.02$ to $g=0.08$. As compared to slow-growing cells, fast-growing cells are much larger and their cell size control is correspondingly weaker. A
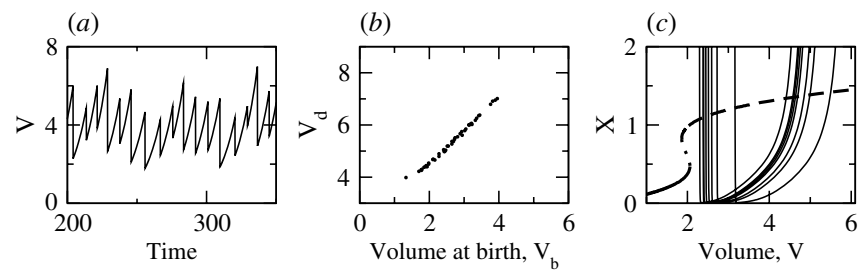

Figure 4. A weak cell size control at fast cell growth rates. Results from simulations of cell proliferation. The model parameters are $g=0.08, s_{x, 1}=0, s_{x, 2}=0.1, a_{x}=1.8, c_{v, d}=0.2$. (a) Plots of the cell volume over successive generation times. (b) Plots of the cell volume at division as a function of the cell volume at birth for 100 cells. (c) Change in the concentration of the $X$-components in a few cells as a function of the cell volume $V$ (thin lines), as compared to their steady state concentration, $X_{0}$ (computed at fixed volumes). The steady states can be stable $(-)$, unstable $(---)$ or a saddle $(\ldots .$.$) . The CC dynamics of cells operates in the limit cycle$ regime which account for the observed weak cell size control.
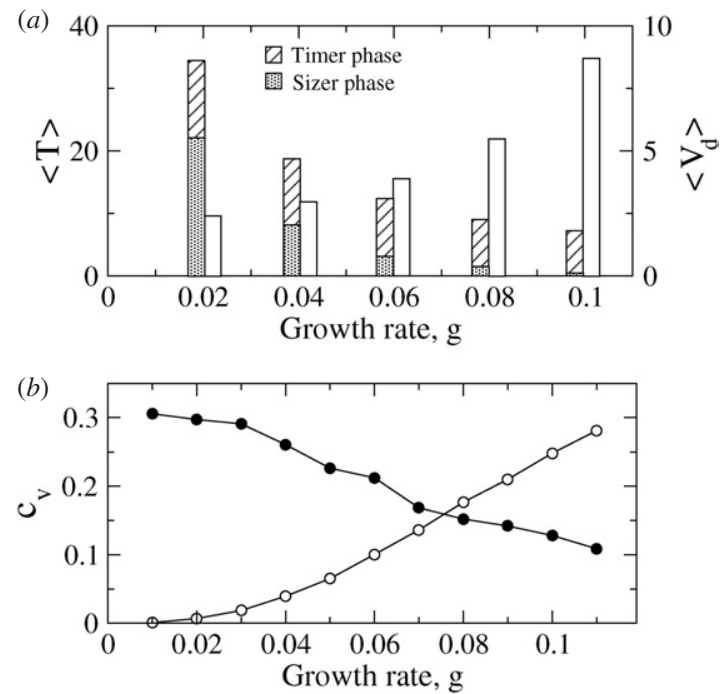

Figure 5. The effects of varying the cell growth rate on cell size control. Results from simulation of cell proliferation where parameters of CC dynamics are the same as in figure 4. (a) Left bars represent the change in the mean interdivision times $\langle T\rangle$ with the respective contribution of the sizer phase (dots) and the timer phase (lines). Right empty bars represent the change in the mean cell volume at division $\left\langle V_{d}\right\rangle$. (b) Change in the coefficient of variation of the cell volume at division $(O)$ and of the interdivision time $(\mathbf{O})$.

strong positive correlation between the cell volumes at division and at birth indeed reflects a weak size control. The situation is reminiscent of that occurring when the $\mathrm{CC}$ dynamics operates in the limit cycle regime during the full cell cycle (compare with figure $3(b))$. In slow growth conditions, the volume is a slow variable as compared to the concentrations of the $\mathrm{CC}$ regulatory components and can be regarded, then, as a bifurcation parameter. This is no longer the case in fast growth conditions in which the cell growth dynamics and $\mathrm{CC}$ progression interfere because of their similar timescales.

In figure 5, the effect of varying the cell growth rate on the cell size control is investigated in a systematic way. Figure 5(a) shows that there is an inverse relationship between the cell growth rates and the interdivision times. The requirement that exponentially growing cells, on average, 
double their mass between birth and division imposes the relation $\langle T\rangle=\ln (2) / g$. Moreover, it is clear that time spent in the sizer phase (i.e., between birth and the CC bifurcation) decays faster than time spent in the timer phase (i.e., between the $\mathrm{CC}$ bifurcation and cell division). Because the timer phase has an intrinsic timescale determined by the limit cycle period of the underlying CC dynamics, the sizer phase is preferentially reduced to match the interdivision time with the doubling mass time, whereby cell size homeostasis is maintained. As a result, the mean cell volume at division increases with the cell growth rate. In the slow growth regime, the cell volume growth during the timer phase is negligible so that the cell volume at division is nearly equal to the cell volume at which the $\mathrm{CC}$ bifurcation occurs. However, as the cell growth rate increases, cell volume expansion during the timer phase itself augments and its contribution to the cell volume at division becomes increasingly large.

The increase in the cell growth rate reduces the relative time spent in the sizer phase and in the timer phase. This suggests that the regulation of cell size homeostasis becomes less and less efficient with increasing growth rates. This is corroborated by the data presented in figure $5(b)$ showing that $c_{v}$ of the cell volume at division and of the interdivision time increases and decreases, respectively, with increasing growth rates. Further, the cell size control breaks down when the cell growth rate becomes larger than 0.11 since the cell volume keeps diverging during continuous cell proliferation.

\subsection{A putative mechanism to reinforce cell size control in fast-growing cells}

Although fast-growing cells display larger sizes than slowgrowing cells, the weakening of cell size control at high growth rates, predicted by our model, has not been observed after measurement of the standard deviations of cell division at various growth rates [16]. It appears therefore that living cells would have developed strategies to improve their mechanism of size control under fast-growing conditions. A possible strategy would be to preserve a sizer phase at large growth rates. This could be achieved in our model by adding to $d_{\alpha}$ a new term, that is proportional to the cell growth rate (with a proportionality factor $\beta$ ). Then relation (4) is replaced by

$$
d_{\alpha}=d_{\alpha, 1}+(1+\beta) g, \quad \alpha=(x, y) .
$$

Figure 6 illustrates how the introduction of this term could improve the cell size control in fast-growing cells. An increase in $d_{\alpha}$ modifies the phase diagram of CC dynamics (figure 6(a)): (i) the SNIPER bifurcation occurs for a larger rate of synthesis, $s_{x}$, and (ii) the frequency of the following limit cycle augments faster for a larger degradation rate $d_{\alpha}$. The cell cycle trajectory for different values of $\beta$ and $g$ can be projected on the phase diagram (see lines). In agreement with the data shown in figure 4 , the sizer phase of cells with $\beta=0$ vanishes when $g$ increases from 0.01 to 0.08 (broken lines). In contrast, for $\beta=7$, the sizer phase is preserved when $g$ increases (full lines).

The preservation of a sizer phase at elevated growth rates considerably reduces the coefficient of variation of the cell volume at division in the presence of asymmetric cell
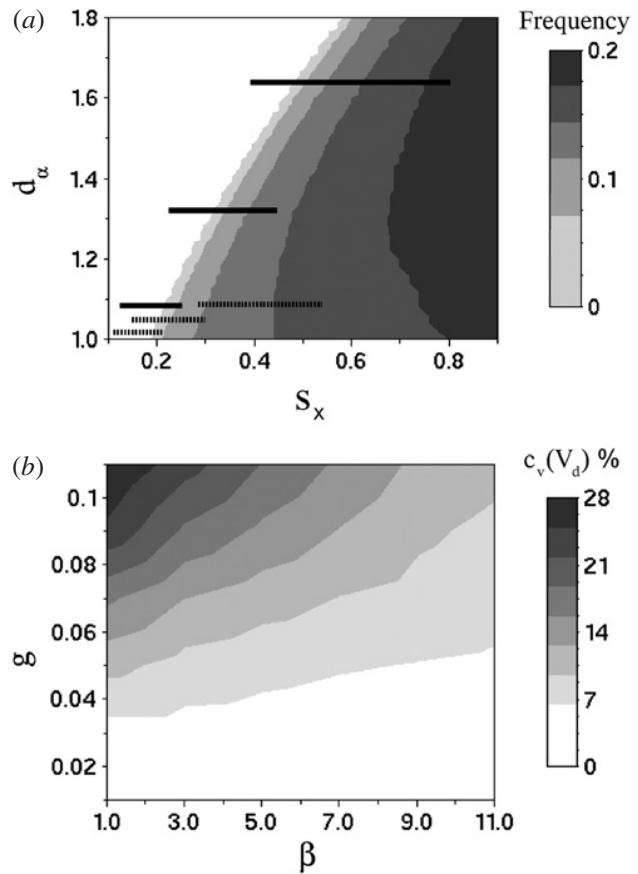

Figure 6. A growth-rate-dependent CC inhibitory activity reinforces the cell size control in fast growing cells. (a) Change in the frequency of the CC oscillatory dynamics as a function of $s_{x}$ and $d_{\alpha}$, which are proportional to the cell volume and the growth rate, respectively. The grayscale denotes the frequency of oscillations within regions of the stable limit cycle. The white region corresponds to the presence of a stable steady state. The lines correspond to the projection of the cell cycle trajectory for different values of $d_{\alpha}$ associated with two values of $\beta$ ( - : $\beta=7 ;---$ : $\beta=0)$ and with three values of the cell growth rate $(g=0.01,0.04$ and 0.08 ). (b) Change in the coefficient of variation of $V_{d}$ (in gray code) as a function of $\beta$ and $g$.

division $\left(c_{v, d}=0.2\right)$ as illustrated in figure $6(b): c_{v}$ decays with increasing values of $\beta$, especially when cells grow rapidly, which underscores that increasing $\beta$ reinforces cell size control.

\subsection{The effect of noises on cell cycle statistical features}

Although there is clear evidence that living cells are equipped with a mechanism enabling them to monitor their size and keep it constant over multiple generations, there is still a significant cell size and interdivision time variation among descendants from the same parent cell. Such variability could have several origins, including the stochasticity in the $\mathrm{CC}$ dynamics, the occurrence of asymmetric cell division and heterogeneity in the cell growth rate. In this section, we investigate how these various sources of noise could influence cell size at division and interdivision times and, more generally, affect cell size homeostasis.

In the following, cell cycle progression is coupled with cell size through $s_{x, 1}=0.1$ and with cell growth, only through the dilution effect $(\beta=0)$. The cell growth rate is assumed to be low enough $(g=0.02)$ such that the cells spend a sufficient time in the sizer phase before they commit to divide. Numerical simulations are performed over 10000 cell cycles in the presence of various sources of noise. These simulations 
(a) $\mathrm{c}_{\mathrm{v}, \mathrm{d}}=0.2$

(i)

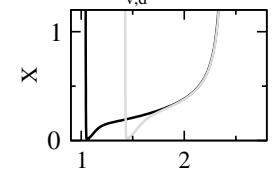

(ii)

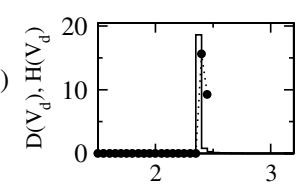

(iii)

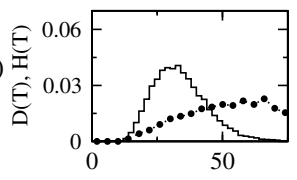

(b) $\sigma_{\mathrm{g}}=0.005$
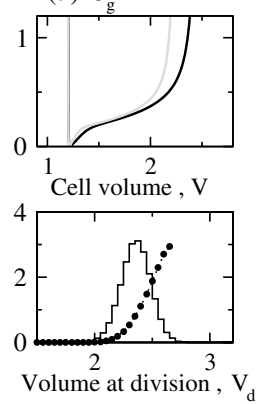

Volume at division, $\mathrm{V}$

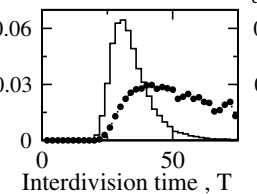

(c) $\eta=200$
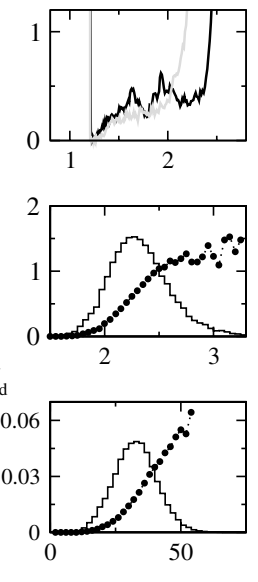

Figure 7. The effect of various sources of noise on the cell cycle variability. Simulations of cell proliferation were performed in the presence of one specific source of noise while other sources were set to zero. The mean cell growth rate is $g=0.02$. (a) A random asymmetry in cell division with $c_{v, d}=0.2$. (b) A growth rate heterogeneity among cells with $\sigma_{g}=0.005$. (c) Noises in the dynamics of $X-/ Y$-components with $\eta=200$. For each type of noise are plotted: the concentration of the $X$-components as a function of the cell volume for two daughter cells (upper panels); the probability density function, $D(x)$ (stairs), and the hazard rate function, $H(x)$

(๑), of the cell volume at division $V_{d}$ (middle panels); the probability density function, $D(x)$ (stairs), and the hazard rate function, $H(x)(\bullet)$, of the interdivision times, $T$ (lower panels).

allow us to compute the probability density function $D(x)$ of the cell volume at division $\left(x=V_{d}\right)$ and of the interdivision time $(x=T)$ and, then, to derive the hazard rate function $H(x)$, which is equal to the ratio of the probability density function to the survival function (i.e., the probability that a cell with a volume or interdivision time $u>x$ would divide):

$$
H(x)=D(x) /\left(1-\int_{0}^{x} D(u) \mathrm{d} u\right) .
$$

The use of the hazard rate function to describe variable distributions is convenient to detect exponential-like decays on the right side of the distribution $D(x)$, for which it is constant.

Figure 7(a) depicts the impact of a random asymmetry in cell division with $c_{v, d}$ of 0.2 . Even though their birth size differs, the two sister cells divide at the same size (figure 7(a)i). Therefore, the distribution of the cell volume at division is very sharp $\left(c_{v} \approx 0.01\right)$ (figure 7(a)ii). Conversely, since newborn cells of different sizes divide at the same size, the interdivision time distribution is broad $\left(c_{v} \approx 0.28\right)$. Interestingly, we find that the hazard rate function saturates, which indicates an exponential-like distribution for half of the cell population that exhibits the longest interdivision time (figure 7(a)iii). It should be noted that the interdivision time variability stems from the variability of the sizer phase.

Figure $7(b)$ depicts the situation in which the growth rate of individual cells is distributed with $\sigma_{g}=0.005$, which is about $25 \%$ of the mean growth rate [19]. If two equally sized sister cells have different growth rates, the one with the higher rate divides at a larger size (figure $7(b) \mathrm{i}$ ), consistently with the data shown in figure $5(b)$. Therefore, the distribution of the cell volume at division displays a significant coefficient of variation $\left(c_{v} \approx 0.06\right)$ (figure $7(b)$ ii). Since the interdivision time and the growth rate are inversely correlated, the interdivision time displays a broad distribution with $c_{v} \approx 0.26$ (figure $7(b)$ iii). The saturation followed by the slow decrease of the hazard rate function of the interdivision time indicates that the $D(T)$ distribution decays almost exponentially. It is also worthy noting that rising $\beta$ increases the variability of the cell volume at division since the critical value of $s_{x}$ for the CC bifurcation itself increases (data not shown).

The effect of molecular noise within the CC dynamics is presented in figure $7(c)$. The standard deviation depends on the concentration of each species and on the absolute number of molecular components through the parameter $\eta$. We set $\eta$ equal to 200, which corresponds to the number of components whose concentration is equal to unity in a unit cell volume (in our dimensionless units). For such a value, our simulations give a typical number of $X$-components at the CC bifurcation ( $V \sim 2$ and $X \sim 0.5$ ) of the order of 200. In the presence of this molecular noise, the cell volume at which the $X$-components trigger a positive feedback in the underlying $\mathrm{CC}$ dynamics is not the same in two sister cells of equal size at birth (figure 7(c)i). The variation in the cell volume at division $\left(c_{v} \approx 0.12\right)$ is much larger than in the above-described cases of asymmetric cell divisions or heterogeneity in the cell growth rate (for $\beta=0$ ), while the variation in the interdivision time $\left(c_{v} \approx 0.24\right)$ is of the same order. Another specific feature associated with molecular noise is that the hazard rate function of the cell volume at division saturates.

When the three preceding sources of noise are combined, each one contributes to specific facets of the $\mathrm{CC}$ statistics (figure 8). Thus, two sister cells might have been born with a different size following an asymmetric division and, afterwards, their size at the CC bifurcation and at division might have been affected by the occurrence of molecular noise or of dissimilar growth rates (figure $8(a)$ ). The lack of correlation between the cell volumes at division and at birth suggests that the mechanism of the cell size control is still operational (figure $8(b)$ ), which underscores that a wide distribution of cell volume at division does not necessarily imply a deficient cell size control. The distribution of cell volume at division displays an exponential tail, which is corroborated by saturating the hazard rate function (figure $8(c)$ ). In comparison, the distribution of interdivision time is broader $\left(c_{v} \approx 0.45\right.$ instead 0.13 ) but it also exhibits an exponential decay (figure $8(d)$ ). A comparison of figures $8(c)$, (d) and 7 clearly points out that the distribution of cell volume at division in the presence of the three sources of noise mirrors that observed in the presence of molecular noise alone. This is not true, however, for the interdivision time distribution whose shape in the presence of the three sources of noise rather mirrors that observed in the presence of an asymmetric cell division and of heterogeneity in the cell growth rate. It is worth noting at last that the exponential-like decay observed in the probability density distribution of both $V_{d}$ and $T$ is experimentally observed in many measurements of the $\mathrm{CC}$ variability. 

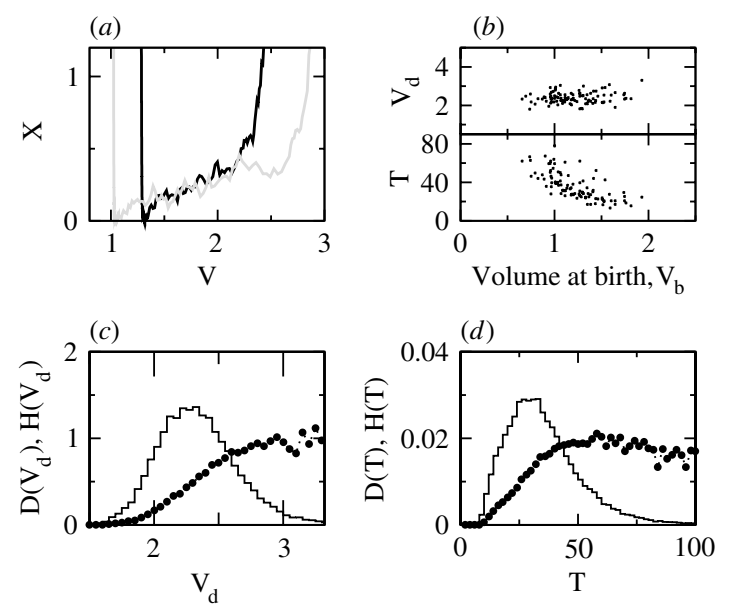

Figure 8. A strong size control in the presence of various sources of noise. Simulations of cell proliferation were performed in the presence of the three sources of noise considered separately in figure 7 (an asymmetric cell division with $c_{v, d}=0.2$; a heterogeneity in the cell growth rate among cells with $\sigma_{g}=0.005$ and noises in the dynamics of $X$-/Y-components with $\eta=200)$. (a) Change in the concentration of the $X$-components as a function of the volume of two daughter cells. (b) Plots of the cell volume at division and interdivision times as a function of the cell volume at birth for 100 cells. $(c),(d)$ Probability density function, $D(x)$ and hazard rate function, $H(x)$, of the cell volume at division, $V_{d}$, and of the interdivision time, $T$.

\section{Discussion}

\subsection{Strong, weak or deficient size control}

The observation that two sister cells born with a different size divide at the same size underscores the existence of a strong cell size control. Expectedly, such a strong cell size control could readily be achieved if cells, during a sizer phase, first grow to a same critical size before they complete events that are required to reach the onset of division, during a timer phase $[3,9]$. The transition between sizer phase and timer phase can be viewed as the result of a bifurcation in the underlying $\mathrm{CC}$ dynamics. For a given cell volume, the $\mathrm{CC}$ dynamics changes from a stable steady state to a stable oscillatory state (i.e. a limit cycle). The occurrence of a stable steady state in the $\mathrm{CC}$ dynamics ensures that cells with heterogeneous states at birth converge to the same volume-dependent steady state before they reach the bifurcation point at a critical cell volume. Either a saddle-node infinite period bifurcation (SNIPER) or a Hopf bifurcation (subcritical or supercritical) may bring about this type of scenario [10, 21].

However, if the CC dynamics is oscillatory, but close to a SNIPER bifurcation, only a weak cell size control can be achieved [24]. Because the period of the limit cycle solution depends on the cell volume, larger cells would tend to have a shorter interdivision time than smaller cells but they would not necessarily divide at the same size, which is indicative of a weak cell size control.

At last, if the period of the limit cycle solution does not depend sufficiently on cell size, as it may occur close to a Hopf bifurcation or far from a SNIPER bifurcation, the cell volume would increase more than two-fold during interdivision time. In this case, cells would suffer a deficient size control and become progressively larger and larger over successive generations.

\subsection{Preserving the cell size checkpoint at a fast growth rate}

When cells grow slowly, a strong size control can operate and the cell volume at the onset of division is slightly larger than at the cell size checkpoint (which marks the transition between the sizer and timer phases). Therefore, the existence of a cell size checkpoint imposes a minimum critical cell size at division when nutrients are scarce [12, 40]. However, even though the existence of a cell size checkpoint clearly prevents the generation of small unviable daughter cells, it is unclear how it would prevent the generation of large, less prolific or unviable cells.

The underlying limit cycle dynamics of the CC model imposes an intrinsic timescale to the timer phase. When the growth rate increases, the cell adjusts the time it needs to double its size with the time required for the $\mathrm{CC}$ dynamics to initiate division by tuning the relative time spent in the sizer and timer phases. As a result, fast-growing cells may spend most, or all, of their cycle time in the timer phase in contrast to slow-growing cells. Therefore, the cell size at division should become larger and the cell size control weaker when the cell growth rate increases. Using a generic eukaryotic model, Csikasz-Nagy and colleagues reached similar conclusions [24]. Our present study extends these findings by proposing a possible strategy to maintain an efficient size control at fast growth rates.

Indeed, although fast-growing cells have been reported to have a larger size than slow-growing cells [3, 11, 12], the measurement of standard deviations of cell division for various growth rates has not demonstrated a failure of cell size control even at elevated growth rates [16]. The strategy that we propose is to add a new $\mathrm{CC}$ inhibitory activity that increases in proportion to the cell growth rate. This new layer of $\mathrm{CC}$ regulation would have, first, to delay the $\mathrm{CC}$ bifurcation such that it would occur at a larger cell volume and, also, to reduce the length of the timer phase. Delaying the $\mathrm{CC}$ bifurcation and modifying the timescale of the $\mathrm{CC}$ dynamics are both simultaneously necessary, which demonstrates that the cell growth rate and the cell size must affect different parameters of the dynamics. This type of $\mathrm{CC}$ regulatory mechanism seems to operate in most eukaryotic cell types. In budding yeast, for instance, a boost in the cell growth rate leads to an increase in volume of the mother cell at bud initiation [40] and a diminution of the duration of the budding phase [14]. We suggest that such growth rate-dependent mechanisms of $\mathrm{CC}$ regulation would be crucial to ensure that daughter cells remain smaller at birth than at CC bifurcation and, then, retain a sizer phase during which they would be able to adjust their size expansion to environmental parameters.

It is noteworthy that, despite the fact that prokaryotes and eukaryotes have selected drastically different strategies to survive in the course of their evolution, their cell cycle processes still share some common features [41-43]. Notably, in E.coli, initiation of DNA replication is tightly regulated 
and its timing relative to that of cell division varies with the cell growth rate. Because the time required for the bacterial chromosome to replicate (around $40 \mathrm{~min}$ ) is independent of the cell growth rate, fast-growing bacteria in which the interdivision time is less than 40 min contain chromosomes with multiple replication forks [1, 44]. Furthermore, the cell size at the onset of DNA replication and the number of chromosome replication origins change discontinuously, but keep a constant ratio when the growth rate increases. A shorter timer phase associated with a larger size at initiation of DNA replication ensures that the cell size at birth is smaller than that at the onset of DNA replication [1] as it occurs in our model.

\subsection{Sloppy but robust size control}

Since cell cycle progression is apparently tightly linked to the cell size, it is puzzling why the cell size at division is so variable, which suggests that the cell size control is a sloppy process. The coefficients of variation of the cell size at division indeed vary between 0.1 and 0.3 depending on the cell type and environmental setting $[15,16]$.

We showed that both heterogeneity in the cell growth rate and molecular fluctuations contribute to the cell size variation although they contribute in a different way. The molecular noise principally affects the cell volume at which the positive feedback of the CC dynamics is activated. Heterogeneity in the cell growth rate, in contrast, can act on both the cell volume at the $\mathrm{CC}$ bifurcation and the length of the timer phase. Together, they induce a sloppy size control by increasing the probability that cells will traverse the cell size checkpoint (before reaching a high plateau) as their size increases [16, 17, 45]. Molecular noise alone fails to account for the peculiar shape of the interdivision time distributions that typically shows an exponential-like tail [16, 18]. The additional presence of heterogeneities in the cell growth rate or/and in the birth volume could account for this facet of the CC statistics as it has been suggested by Koch [46]. It is worthwhile here to mention the results of an experimental study analyzing the effects of various noises and their combined impact on variability in the budding yeast cell cycle [19]. This recent study corroborates some assumptions and results of our modeling paper. Notably, it estimates and emphasizes the respective contributions of the molecular noise and of the variability of cell size at birth on the temporal variability of the cell cycle.

The cell size control mechanism depicted here is operational in the presence of large amounts of noise: a broad distribution of the cell size at division does not necessarily imply the occurrence of a faulty cell size control. This is because the divergent effects of noises that may affect the onset and the length of timer phase in parental cells are balanced by the fact that the $\mathrm{CC}$ trajectories of daughter cells are attracted onto a fixed point during the sizer phase.

\subsection{A minimal modeling approach}

Many existing cell cycle models attempt to build as realistic as possible representations of the intricate $\mathrm{CC}$ regulatory networks that operate in different living systems [47, 49]. A complementary approach is to design minimal mathematical models capable of recapitulating the underlying universal dynamical properties of these networks. Such minimal models are highly useful and relevant for understanding the basic function of more complex networks, as coarsegrained modules in a more encompassing model. From this perspective, we deliberately have made our model dimensionless and as simple as possible.

Regarding the CC dynamics, our analysis is restricted to the dynamics of the module that controls the cell size checkpoint. We assume that the essential features of the dynamics of this module can be captured within a twovariable model based on the dynamics of protein synthesis and degradation. However, the activity of the cyclin-Cdk complexes is limited not only by the rates of cyclin synthesis and degradation, but also by faster phosphorylation and dephosphorylation reactions [34]. It is well acknowledged, for instance, that Cdk inhibitor phosphorylation by cyclin-Cdk complexes plays an essential role in the switch from G1 to $\mathrm{S}$ phase. Our model mimics such a positive feedback (or doublenegative feedback) by attributing an autocatalytic activity to the $X$-components.

Another assumption of the model is that individual cell volume growth occurs exponentially although linear or bilinear growth patterns have been documented in some cases. This assumption, however, is not crucial for the conclusions of the paper. In the case of linear cell growth, for instance, cell size homeostasis would occur even without coupling between growth and division. Nevertheless, the distinction between strong and weak cell size control as well as the role of a growth-rate-dependent $\mathrm{CC}$ inhibitory activity in preserving a robust cell size control at high growth rate would still be fully relevant. In addition, the distributions of cell size at division and of interdivision time are quite similar in the case of linear and exponential growth, except for the interdivision time distribution in the presence of random asymmetric cell division that may significantly change depending on the growth pattern.

At last, specific assumptions regarding the coupling between the CC and cell growth dynamics were made. First, we postulated (as others $[10,24]$ ) that the rate of appearance of the CC components that initiate cell division increases with cell size (see equation (5)). This hypothesis could be justified, for instance, if the rate-limiting step in the appearance of the active cyclin-Cdk complexes was the translocation of the complexes from the cytoplasm to the nucleus. It should be reminded, indeed, that the cyclin-Cdk complexes are formed in the cytoplasm before they are transported to the nucleus where they exert their cell cycle function. In a recent theoretical study, Yang et al [39] argued that the rate of translocation of proteins from the cytoplasm to the nucleus (which depends on the ratio between the cytoplasmic volume and the nuclear surface) could be a cell size-dependent rate-limiting step in the appearance of active cyclin-Cdk complexes in nucleus. Second, we anticipated the existence of a negative regulator of the cyclin-Cdk complexes whose inhibitory activity would increase with the cell growth rate (see equation (9)). This could occur for instance if, with increasing growth rate, the 
ratio between the rates of synthesis and of degradation of such a CC inhibitor would increase while the ratio between the rate of appearance and of disappearance of the cyclinCdk complexes would remain unchanged, or, at least, would increase slower. It is important also to mention that the cyclin$\mathrm{Cdk}$ complexes have a plethora of substrates and that they are also involved in CC-unrelated activities which may compete with their CC-oriented activities [48]. This may be another mechanism through which growth-rate-dependent regulation of the cell cycle might occur.

\section{Conclusion and outlook}

The fact that living cells have the capacity to sustain a constant size over continual proliferation rests upon their capacity to adjust their interdivision time and growth extent until division according to their birth size. In this work, a minimal mathematical model is formulated that depicts how a robust cell size homeostasis can be achieved by intimately connecting cell growth and cell cycle progression before onset of division. A crucial hypothesis of our model is that the cell size and cell growth rate exert an opposite effect on the activation of the positive feedback that triggers the $\mathrm{CC}$ bifurcation that ultimately culminates in cell division: cell size acts as a promoter and the cell growth rate as an antagonist.

In the eukaryotic $\mathrm{CC}, \mathrm{CC}$ bifurcation (i.e. the cell size checkpoint) is usually associated with the G1/S or the G2/M transition. One key question now is what and how biochemical activities connected to the cell size and the cell growth rate regulate the positive feedback that propels cells into the $\mathrm{S}$ or $\mathrm{M}$ phase. Conceivably, regulation of traffic of the $\mathrm{CC}$ regulatory components from the cytoplasm (the site of synthesis) to the nucleus (the site of action) could play a critical role in setting the cell size at which the $\mathrm{S}$ or $\mathrm{M}$ phase is initiated [39, 49-51]. On the other hand, recent experiments suggest that growthrate-dependent regulation of the cell cycle may take root in the versatile connection between ribosomal biogenesis and replication initiators [52-56]. Expectedly, each species must have evolved a unique wiring diagram between its growth and $\mathrm{CC}$ regulatory pathways to improve its adaptation and, thereby, its long-term survival chances facing changeable environments. Tremendous efforts, thus, are still to be made to dissect such wiring diagrams in the different constitutive cells of each living species.

\section{Acknowledgments}

The authors are grateful to Dr Thérèse David-Pfeuty for stimulating discussions and her critical reading of the manuscript. One of the authors (BP) is supported by the Japanese Society for the Promotion of Science (JSPS).

\section{References}

[1] Donachie W D 1968 Relationship between cell size and time of initiation of DNA replication Nature 219 1077-8

[2] Johnston G C, Pringle J R and Hartwell L H 1977 Coordination of growth with cell division in the yeast Saccharomyces cerevisiae Exp. Cell Res. 105 79-98
[3] Fantes P A 1977 Control of cell size and cycle time in Schizosaccharomyces pombe J. Cell Sci. 24 51-67

[4] Masui Y and Wang P 1998 Cell cycle transition in early embryonic development of Xenopus laevis Biol. Cell $90537-48$

[5] Prescott D M 1956 Relation between cell growth and cell division: II. The effect of cell size on cell growth rate and generation time in Amoeba proteus Exp. Cell Res. 11 86-94

[6] Donnan L and John P C 1983 Cell cycle control by timer and sizer in Chlamydomonas Nature 304 630-3

[7] Yen A, Fried J, Kitahara T, Stride A and Clarkson B D 1975 The kinetic significance of cell size, I. Variation of cell cycle parameters with size measured at mitosis Exp. Cell Res. 95 295-302

[8] Jorgensen P and Tyers M 2004 How cell coordinate growth and division Curr. Biol. 14 1014-27

[9] Sveiczer A, Novak B and Mitchison J M 1996 The size control of fission yeast revisited J. Cell Sci. 109 2947-57

[10] Qu Z, MacLellan W R and Weiss J N 2003 Dynamics of the cell cycle: checkpoints, sizers, and timers Biophys. J. 85 3600-11

[11] Schaechter M, Maaloe O and Kjeldgaard N O 1958 Dependency on medium and temperature of cell size and chemical composition during balanced grown of Salmonella typhimurium J. Gen. Microbiol. 19 592-606

[12] Shehata T E and Marr A G 1971 Effect of nutrient concentration on the growth of Escherichia coli J. Bacteriol. $107210-6$

[13] Fantes P and Nurse P 1977 Control of cell size at division in fission yeast by a growth-modulated size control over nuclear division Exp. Cell Res. 107 377-86

[14] Tyson C B, Lord P G and Wheals A E 1979 Dependency of size of Saccharomyces cerevisiae cells on growth rate J. Bacteriol. 138 92-8

[15] Anderson E C, Bell G I, Petersen D F and Tobey R A 1969 Cell growth and division: IV. Determination of volume growth rate and division probability Biophys. J. 9 246-63

[16] Lord P G and Wheals A E 1981 Variability in individual cell cycles of Saccharomyces cerevisiae J. Cell Sci. 50 361-76

[17] Wheals A E 1982 Size control models of Saccharomyces cerevisiae cell proliferation Mol. Cell Biol. 2 361-8

[18] Shields R, Brooks R F, Riddle P N, Capellaro D F and Delia D 1978 Cell size, cell cycle and transition probability in mouse fibroblasts Cell 15 469-74

[19] Di Talia S, Skotheim J M, Bean J M, Siggia E D and Cross F R 2007 The effects of molecular noise and size control on variability in the budding yeast cell cycle Nature 448 947-51

[20] Hartwell L H and Unger U 1977 Unequal division in Saccharomyces cerevisiae and its implications for the control of cell division J. Cell Biol. 75 422-35

[21] Tyson J J, Chen K and Novak B 2001 Network dynamics and cell physiology Nat. Rev. Mol. Cell Biol. 2 908-16

[22] Tyson J J, Csikasz-Nagy A and Novak B 2002 The dynamics of cell cycle regulation Bioessays 24 1095-109

[23] Ingolia N T and Murray A 2004 The ups and downs of modeling the cell cycle Curr. Biol. 14 771-7

[24] Csikasz-Nagy A, Battagtokh D, Chen K C, Novak B and Tyson J J 2006 Analysis of a generic model of eukaryotic cell-cycle regulation Biophys. J. 90 4361-79

[25] Sveiczer A, Tyson J J and Novak B 2001 A stochastic, molecular model of the fission yeast cell cycle: role of the nucleocytoplasmic ratio in cycle time regulation Biophys. Chem. 92 1-15

[26] Mitchison J M 1957 The growth of single cells: I. Schizosaccharomyces pombe Exp. Cell Res. 13 244-62

[27] Buchwald P and Sveiczer A 2006 The time-profile of cell growth in fission yeast: model selection criteria favoring 
bilinear models over exponential ones Theor. Biol. Med. Model. 27 3-16

[28] Cooper S 2006 Distinguishing between linear and exponential cell growth during the division cycle: single-cell studies, cell-culture studies, and the object of cell-cycle research Theor. Biol. Med. Model. 23 3-10

[29] Woldringh C L, Huls P G and Vischer N O 1993 Volume growth of daughter and parent cells during the cell cycle of Saccharomyces cerevisiae a/alpha as determined by image cytometry J. Bacteriol. 175 3174-81

[30] Alberghina L, Smeraldi C, Ranzi B M and Porro D 1998 Control by nutrients of growth and cell cycle progression in budding yeast, analyzed by double-tag flow cytometry J. Bacteriol. 180 3864-72

[31] Tyson J J and Novak B 2001 Regulation of the eukaryotic cell cycle: molecular antagonism, hysteresis, and irreversible transitions J. Theor. Biol. 210 249-63

[32] Pomerening J R, Sontag E D and Ferrell J E Jr 2003 Building a cell cycle oscillator: hysteresis and bistability in the activation of Cdc2 Nat. Cell Biol. 5 346-51

[33] Han Z, Yang L, MacLellan W R, Weiss J N and Qu Z 2005 Hysteresis and cell cycle transitions: how crucial is it? Biophys. J. 88 1626-34

[34] Morgan D O 1997 Cyclin-dependent kinases: engines, clocks, and microprocessors Annu. Rev. Cell Dev. Biol. 13 261-91

[35] Fang G, Yu H and Kirschner M W 1999 Control of mitotic transitions by the anaphase-promoting complex Phil. Trans. R. Soc. B 354 1583-90

[36] Tyson J J, Chen K C and Novak B 2003 Sniffers, buzzers, toggles and blinkers: dynamics of regulatory and signaling pathways in the cell Curr. Opin. Cell Biol. 15 221-31

[37] Gillespie D T 2000 The chemical Langevin equation J. Chem. Phys. 113 197-306

[38] Moore J D, Yang J, Truant R and Kornbluth S 1999 Nuclear import of Cdk/cyclin complexes: identification of distinct mechanisms for import of $\mathrm{Cdk} 2 /$ cyclin $\mathrm{E}$ and $\mathrm{Cdc} 2 /$ cyclin B1 J. Cell Biol. 144 213-24

[39] Yang L, Han Z, Robb MacLellan W, Weiss J N and Qu Z 2005 Linking cell division to cell growth in a spatiotemporal model of the cell cycle J. Theor. Biol. 241 120-33

[40] Johnston G C, Ehrhardt C W, Lorincz A and Carter B L 1979 Regulation of cell size in the yeast Saccharomyces cerevisiae J. Bacteriol. 137 1-5
[41] Nasmyth K 1995 Evolution of the cell cycle Phil. Trans. R. Soc. B 349 271-81

[42] Margolin W and Bernander R 2004 How do prokaryotic cells cycle? Curr. Biol. 14 768-70

[43] Brazhnik P and Tyson J J 2006 Cell cycle control in bacteria and yeast: a case of convergent evolution? Cell Cycle 5 522-9

[44] Cooper S and Helmstetter C E 1968 Chromosome replication and the division cycle of Escherichia coli B/r J. Mol. Biol. 31 519-40

[45] Tyson J J and Diekmann O 1986 Sloppy size control of the cell division cycle $J$. Theor. Biol. 118 405-26

[46] Koch A L 1980 Does the variability of the cell cycle result from one or many chance events? Nature 286 80-2

[47] Chen K C, Calzone L, Csikasz-Nagy A, Cross F R, Novak B and Tyson J J 2004 Integrative analysis of cell cycle control in budding yeast $\mathrm{Mol}$. Biol. Cell 15 3841-62

[48] David-Pfeuty T, Nouvian-Dooghe Y, Sirri V, Roussel P and Hernandez-Verdun D 2001 Common and reversible regulation of wild-type p53 function and of ribosomal biogenesis by protein kinases in human cells Oncogene 20 5951-63

[49] Barberis M, Klipp E, Vanoni M and Alberghina L 2007 Cell size at $\mathrm{S}$ phase initiation: an emergent property of the $\mathrm{G} 1 / \mathrm{S}$ network PLoS Comput. Biol. 3 649-66

[50] Futcher B 1996 Cyclins and the wiring of the yeast cell cycle Yeast 12 1635-46

[51] Umen J G 2005 The elusive sizer Curr. Opin. Cell Biol. 17 435-41

[52] Montagne J, Stewart M J, Stocker H, Hafen E, Kozma S C and Thomas G 1999 Drosophila S6 kinase: a regulator of cell size Science $2852126-9$

[53] Jorgensen P, Nishikawa J L, Breitkreutz B J and Tyers M 2002 Systematic identification of pathways that couple cell growth and division in yeast Science 297 395-400

[54] Jorgensen P, Rupes I, Sharom J R, Schneper L, Broach J R and Tyers M 2004 A dynamic transcriptional network communicates growth potential to ribosome synthesis and critical cell size Genes Dev. 18 2491-505

[55] Dez C and Tollervey D 2004 Ribosome synthesis meets the cell cycle Curr. Opin. Microbiol. 7 631-7

[56] Bernstein K A, Bleichert F, Bean J M, Cross F R and Baserga S J 2007 Ribosome biogenesis is sensed at the start cell cycle checkpoint Mol. Biol. Cell 18 953-64 